\title{
Massive stars and the creation of our Galactic Center
}

\author{
Donald F. Figer \\ Space Telescope Science Institute, \\ 3700 San Martin Drive, Baltimore, MD 21218, USA \\ and \\ Department of Physics and Astronomy, Johns Hopkins University, \\ Charles and 34th Street, Baltimore, MD 21218, USA
}

\begin{abstract}
Our Galactic Center hosts over $10 \%$ of the known massive stars in the Galaxy. The majority of these stars are located in three particularly massive clusters that formed within the past $5 \mathrm{Myr}$. While these clusters are extraordinary, their formation repesents about half of the total inferred starformation rate in the Galactic Center. There is mounting evidence that the clusters are just present-day examples of the hundreds of such similar clusters that must have been created in the past, and whose stars now comprise the bulk of all stars seen in the region. I discuss the massive stellar content in the Galactic Center and present a new analysis that suggests that effects of continuous star-formation in the Galactic Center can be seen in the observed luminosity functions newly-obtained HST-NICMOS and Gemini-AO data.
\end{abstract}

\section{Introduction}

Over $10 \%$ of the known massive stars $\left(M_{\mathrm{i}}>20 \mathrm{M}_{\odot}\right)$ in the Galaxy reside in three clusters of young stars located within $30 \mathrm{pc}$ of the Galactic Center (GC). These clusters are the most massive young clusters in the Galaxy and contain approximately 30 Wolf-Rayet (WR) stars (see the Galactic WR catalogue of van der Hucht 2001), at least two Luminous Blue Variables (LBV), approximately a half dozen red supergiants, and about 300 O-type stars. Together, they emit enough ionizing radiation to account for roughly half of the thermal radio emission in the central few degrees of the Galaxy, suggesting that the young clusters contain approximately half of the stars recently formed in this region. An additional collection of young stars exists in the region, with members scattered about the central $50 \mathrm{pc}$; some have evolved to the WR stage, while others are still deeply embedded within their natal dust cocoons.

The current star-formation rate can be approximated by dividing the mass in newly formed stars by their ages, i.e., $5 \times 10^{4} \mathrm{M}_{\odot} / 5 \mathrm{Myr} \simeq 0.01 \mathrm{M}_{\odot} \mathrm{yr}^{-1}$, or a star-formation rate density of $10^{-7} \mathrm{M}_{\odot} \mathrm{yr}^{-1} \mathrm{pc}^{-3}$. This rate is approximately 250 times higher than the mean rate in the Galaxy, and about the same factor lower than the rate in starburst galaxies. Clearly, the Galactic Center has formed a plethora of stars in the past $5 \mathrm{Myr}$, but it is less apparent when the millions of stars in the central $50 \mathrm{pc}$ formed. If we assume that the star-formation rate in the past was similar to the present rate, then the total mass of stars formed over the past $10 \mathrm{Gyr}$ is $\sim 10^{8} \mathrm{M}_{\odot}$ within a radius of $30 \mathrm{pc}$ of the Galactic Center, or an 
order of magnitude greater than this amount over the whole Central Molecular Zone, as first suggested by Serabyn \& Morris (1996).

This review summarizes the current state of knowledge concerning the massive stars in the Galactic Center, and the history of star-formation therein.

\section{The Central Cluster}

The first young stars discovered in the GC are within the central parsec of the Galaxy (Becklin \& Neugebauer 1968). Rieke \& Lebofsky (1982) and Lebofsky, Rieke \& Tokunaga (1982) confirmed the identification of blue and red supergiants in the region, claiming that these stars formed in a burst of star-formation $\sim 1$ Myr ago and that their presence could account for the nearby ionized gas and heated dust. Forrest et al. (1987) discovered a blue supergiant in the center having a broad Brackett- $\alpha$ emission line, and Allen, Hyland \& Hillier (1990) identified this same star as having a spectrum similar to those for evolved massive stars in the Magellanic Clouds. Further studies discovered that many of the blue supergiants are evolved massive stars with spectra having prominent $\mathrm{He} I$ emission lines, firmly establishing a starburst event roughly $5 \mathrm{Myr}$ ago (Krabbe et al. 1991, 1995; Allen 1994; Rieke \& Rieke 1994; Blum et al. 1995; Eckart et al. 1995; Genzel et al. 1996; Tamblyn et al. 1996).

We now know that the Central Cluster contains over 30 evolved massive stars having $M_{\mathrm{i}}>20 \mathrm{M}_{\odot}$. A current estimate of the young population includes 9 WR stars, 20 stars with Ofpe/WN9-like $K$-band spectra, several red supergiants, and many luminous mid-infrared sources in a region of $1.6 \mathrm{pc}$ in diameter centered on Sgr A* (Genzel et al. 1996). In addition, I estimate that it contains $\sim 100$ O-type stars (O7 and later) still on the main sequence. Najarro et al. (1994) modeled the infrared spectrum of the AF star finding that it is a helium-rich blue supergiant/Wolf-Rayet star, characterized by a strong stellar wind and a moderate amount of Lyman ionizing photons. Najarro et al. (1997) expanded this work by analyzing spectra of eight blue supergiants in the center, finding extremely strong stellar winds $\left(\dot{M} \approx 5-80 \times 10^{-5} \mathrm{M}_{\odot} \mathrm{yr}^{-1}\right)$, relatively small outflow velocities $\left(v_{\infty} \approx 300-1000 \mathrm{~km} \mathrm{~s}^{-1}\right)$, effective temperatures from $17000 \mathrm{~K}$ to $30000 \mathrm{~K}$, stellar luminosities of $1-30 \times 10^{5} \mathrm{~L}_{\odot}$, and spectral characteristics consistent with an Ofpe/WN9 classification. They concluded that the $\mathrm{He}$ I emission line stars power the central parsec and belong to a young stellar cluster of massive stars which formed a few million years ago. More recently, Paumard et al. (2001) reviewed the emission-line stellar population in the central parsec, using new narrow-band infrared imaging. They found that the brightest emission-line stars divide into two categories, bright narrow-line $\left(200 \mathrm{~km} \mathrm{~s}^{-1}\right)$ and faint broad-line $\left(1000 \mathrm{~km} \mathrm{~s}^{-1}\right)$ stars, the former being clustered within a few arcseconds of Sgr A*, and the latter being distributed between $5^{\prime \prime}$ and $10^{\prime \prime}$ from the center.

Eckart et al. (1999) and Figer et al. (2000) identified massive stars within a few AU of the supermassive black hole. Evidently, a significant fraction of this small group of stars are young $\left(t_{\text {age }}<20 \mathrm{Myr}\right)$ and require extraordinarily dense pre-collapse cores $\left(\rho>10^{11} \mathrm{~cm}^{-3}\right)$ or formation sites much further away from the central black hole than their present location would suggest. Early results from proper-motion studies suggest that at least some of the stars in this cluster are 


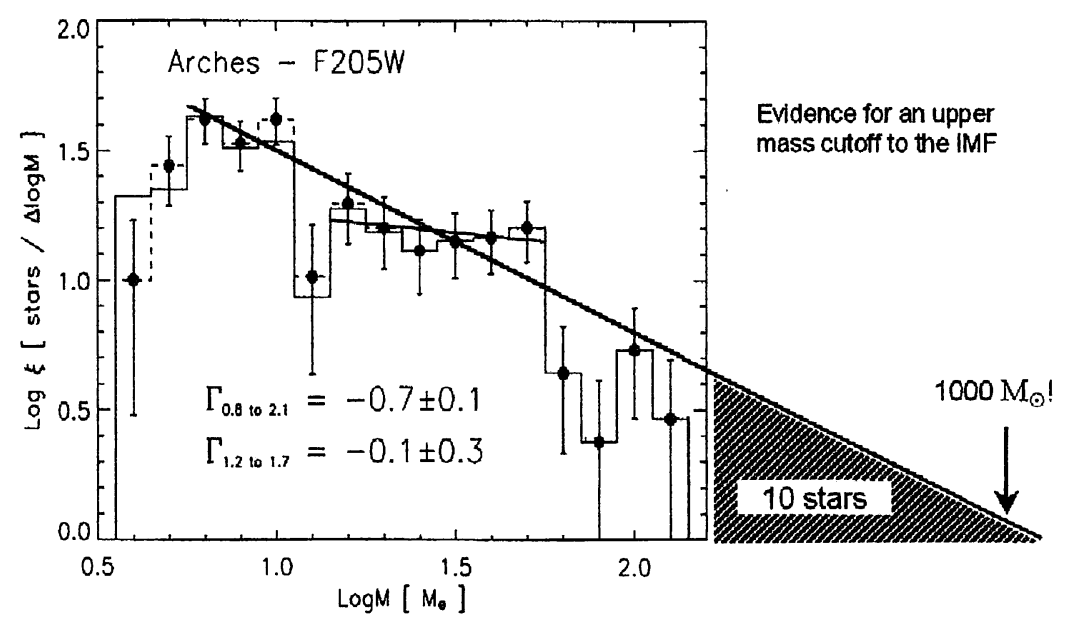

Figure 1. Mass function in the Arches Cluster. Note the absence of stars more massive than $M_{\mathrm{i}}>300 \mathrm{M}_{\odot}$, implying the detection of an upper mass cutoff.

bound to the black hole and are not on highly elliptical orbits (Ghez et al. 2001; Eckart et al. 2002); therefore they are likely to be near to their formation sites.

\section{The Quintuplet Cluster}

The Quintuplet Cluster is, in projection, located $\sim 30 \mathrm{pc}$ NE of the Galactic Center (Glass, Catchpole \& Whitelock 1987). In addition to the five bright stars for which the Quintuplet was named (Nagata et al. 1990; Okuda et al. 1990), the Quintuplet Cluster contains a variety of massive stars, including four WN, five WC (possibly ten, see below), two WN9/Ofpe, two LBV, one red supergiant and several dozen less-evolved blue supergiants (Figer et al. 1999a, 1999c). The five Quintuplet-proper members are massive stars $\left(L \approx 10^{5} \mathrm{~L}_{\odot}\right)$ embedded within dusty cocoons, although their spectral types and evolutionary status are unknown (Moneti et al. 2001). Figer et al. $(1996,1999$ a) argue that these stars are dust-enshrouded WCL stars, similar to WR 104 and WR 98a (Williams et al. 1987; Williams et al. these Proceedings; Tuthill et al. 1999; Tuthill et al. these Proceedings). In addition to these post-main sequence stars, it is likely that some 100 O-type stars still on the main sequence exist in the cluster, assuming a flat to Salpeter IMF. The total cluster mass is estimated to be $\sim 10^{4} \mathrm{M}_{\odot}$; of this, the stars with certain spectral identifications, i.e., the most massive ones, contribute a few $10^{3} \mathrm{M}_{\odot}$. Given the extended distribution of the cluster, the implied mass density is greater than few thousand $\mathrm{M}_{\odot} \mathrm{pc}^{-3}$. The total ionizing flux is $\sim 10^{51} \mathrm{ph} \mathrm{s}^{-1}$, enough to ionize the nearby 'Sickle' H II region (G 0.18-0.04). The total luminosity from the massive cluster stars is $\sim 10^{7.5} \mathrm{~L}_{\odot}$, enough to account for the heating of the nearby molecular cloud, M $0.20-0.033$.

The two LBVs are added to the list of six LBVs in the Galaxy. They include the Pistol Star (Moneti et al. 1994; Figer 1995; Figer et al. 1995, 1995b, 1998, 1999b; Cotera 1995; Cotera et al. 1996), one of the most luminous stars known, 

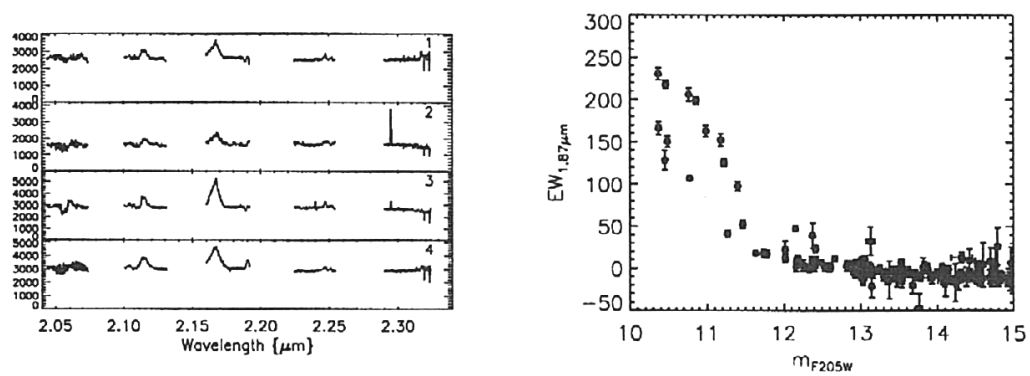

Figure 2. Left: $K$-band spectra of four Wolf-Rayet stars (WNL) in the Arches Cluster, from Figer et al. (2002). Emission lines can be seen at He I $2.058 \mu \mathrm{m}, \mathrm{N}$ III $2.104 \mu \mathrm{m}$, He I 2.112-113 $\mu \mathrm{m}, \mathrm{N}$ III $2.115 \mu \mathrm{m}$, Her $/ \mathrm{H}$ I $2.166 \mu \mathrm{m}$, He I $2.189 \mu \mathrm{m}$, and N III $2.224-225 \mu \mathrm{m}$. The sharp feature near $2.32 \mu \mathrm{m}$ in the spectrum for star \#2 is due to a detector defect, and the absorption features longward of $2.33 \mu \mathrm{m}$ are due to imperfect correction for telluric absorption. Right: Equivalent width of the $1.87 \mu \mathrm{m}$ feature in massive stars of the Arches Cluster (Figer et al. 2002; see also Blum et al. 2001) as a function of apparent magnitude in the HST-NICMOS F205W filter. The feature has contributions from $\mathrm{He} \mathrm{I}$ and $\mathrm{HI}$.

and a newly identified LBV (FMM 362, Geballe et al. 2000) that is nearly as luminous as the Pistol Star. Both stars are luminous, 'blue', and variable, and the Pistol Star has ejected $10 \mathrm{M}_{\odot}$ of material in the past $\sim 10^{4} \mathrm{yr}$, as evidenced by the remarkable Pistol nebula of ionized gas surrounding the star (Figer et al. 1999b; Moneti et al. 2001). Most of the luminous stars in the cluster are thought to be 3-5 Myr old, but significant age differences remain, i.e., the Pistol Star is thought to be $\sim 2$ Myr old.

\section{The Arches Cluster}

First discovered about 10 years ago as a compact collection of a dozen or so emission-line stars (Cotera et al. 1992; Nagata et al. 1995; Figer 1995; Cotera 1995; Cotera et al. 1996; Blum et al. 2001), the Arches Cluster contains thousands of stars, including at least 160 O-type stars, according to Figer et al. (1999c). Figer et al. (1999c) used HST-NICMOS observations to estimate a total cluster mass $\left(Z 10^{4} \mathrm{M}_{\odot}\right)$ and radius $(0.2 \mathrm{pc})$ to arrive at an average mass density of $3 \times 10^{5} \mathrm{M}_{\odot} \mathrm{pc}^{-3}$ in stars, suggesting that the Arches Cluster is the densest, and one of the most massive, young clusters in the Galaxy. They further used these data to estimate an initial mass function (IMF) which is relatively flat $(\Gamma \simeq-$ $0.6 \pm 0.1)$ with respect to what has been found for the solar neighborhood $(\Gamma \simeq-$ 1.35, Salpeter 1955) and other Galactic clusters (Scalo 1998). Stolte et al. (2002) recently confirmed this flat slope by analyzing the same data and recently obtained Gemini-AO data. Figer et al. (2002) estimated an age of $2.5 \pm 0.5 \mathrm{Myr}$, based on the magnitudes, colors, mix of spectral types, and quantitative spectral analysis of stars in the cluster. Given the current state of knowledge about this cluster, it now seems apparent that we have observed a firm upper-mass cutoff, as shown in Figure 1. Note that we should expect at least 10 stars more massive than $M_{\mathrm{i}}=300 \mathrm{M}_{\odot}$. Indeed, we should even expect one star with an ini- 


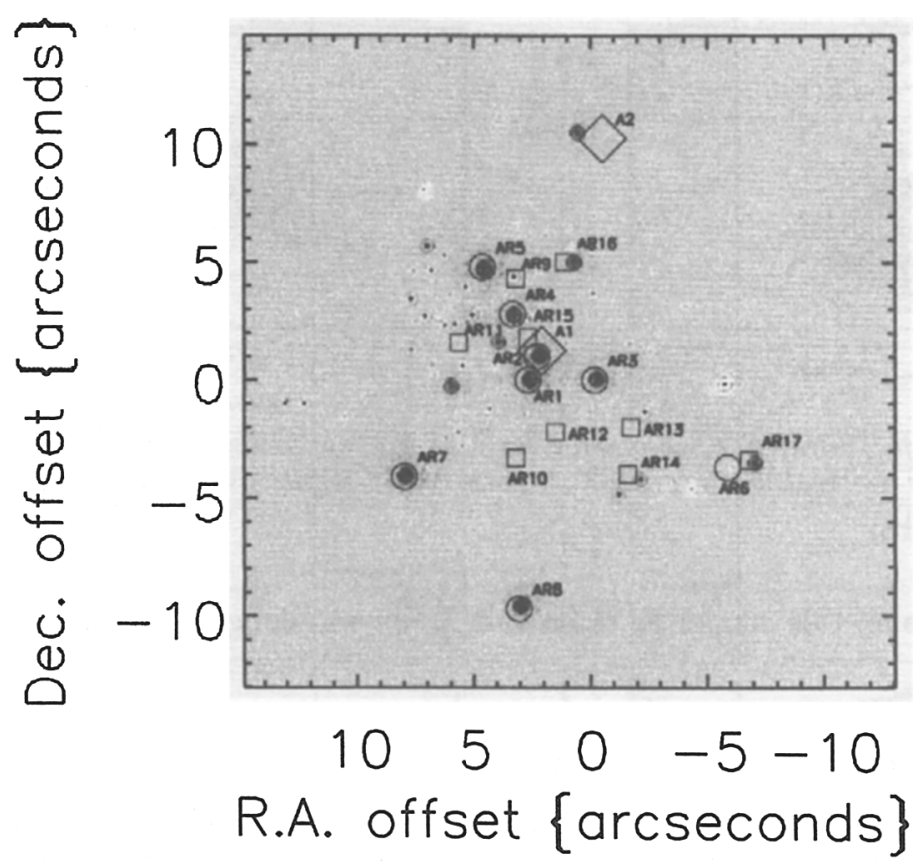

Figure 3. Difference image, F187N-F190N, highlighting stars with excess emission at $1.87 \mu \mathrm{m}$. Radio sources $(\nu=4.9 \mathrm{GHz})$ are shown by circles, as identified in Lang et al. (2001), and as squares, as identified by Figer et al. (2002). X-ray sources are shown by diamonds, as identified by Yusef-Zadeh et al. (2002).

tial mass of $1000 \mathrm{M}_{\odot}$ ! Of course, it is questionable how long such a star would survive; however, it is clear that the Arches Cluster IMF cuts off at around $150 \mathrm{M}_{\odot}$. Finally, even if we steepen the IMF slope to the Salpeter value, we still should expect at least four stars more massive than $300 \mathrm{M}_{\odot}$.

Figer et al. (2002) conclude that the most massive stars are bona-fide WolfRayet stars and are some of the most massive stars known, having $M_{\mathbf{i}}>100 \mathrm{M}_{\odot}$, and prodigious winds, $\dot{M}>10^{-5} \mathrm{M}_{\odot} \mathrm{yr}^{-1}$, that are enriched with helium and nitrogen. These findings are largely based upon the spectra and narrow-band equivalent widths shown in Figure 2, and a detailed quantitative analysis of these data. Figer et al. (2002) found an upper limit to the velocity dispersion of $22 \mathrm{~km} \mathrm{~s}^{-1}$, implying an upper limit to the cluster mass of $7 \times 10^{4} \mathrm{M}_{\odot}$ within a radius of $0.23 \mathrm{pc}$, and a bulk velocity of $v_{\text {cluster }} \simeq+55 \mathrm{~km} \mathrm{~s}^{-1}$ for the cluster. It appears that the cluster happens to be ionizing, and approaching, the surface of a background molecular cloud, thus producing the Thermal Arched Filaments. They estimate that the cluster produces $4 \times 10^{51}$ ionizing $\mathrm{ph} \mathrm{s}^{-1}$, more than enough to account for the observed thermal radio flux from the nearby cloud. Commensurately, it produces $10^{7.8} \mathrm{~L}_{\odot}$ in total luminosity, providing the heating source for the nearby molecular cloud, $L_{\text {cloud }} \approx 10^{7} \mathrm{~L}_{\odot}$. These interactions between a cluster of hot stars and a wayward molecular cloud are similar to those seen in the 'Quintuplet-Sickle' region. Finally, note that significant 

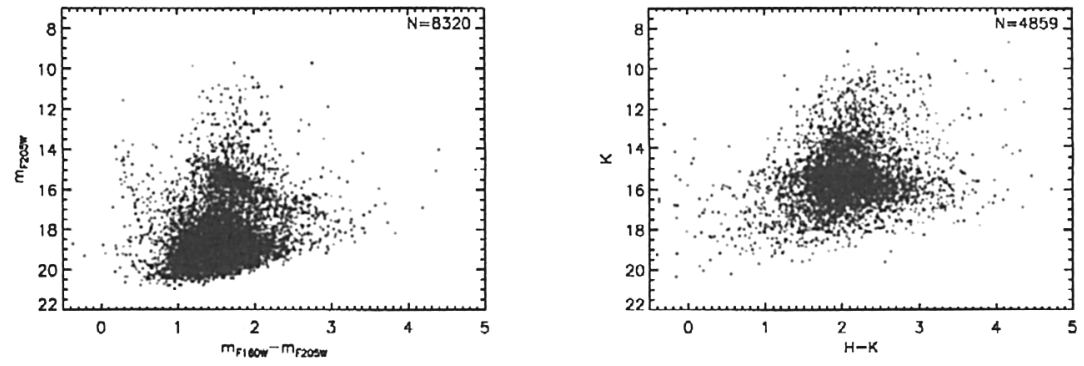

Figure 4. Left: Infrared color-magnitude diagram for fields stars in the Galactic Center as obtained from HST-NICMOS data. Right: Idem, but from Gemini-AO data.

work is being done on this cluster at radio and X-ray wavelengths, as shown in Figure 3.

\section{The star-formation history of the Galactic Center}

The evidence for recent ( $<10 \mathrm{Myr})$ star-formation in the Galactic Center abounds. The Lyman continuum flux emitted in the central few degrees of the Galaxy is about $10^{52} \mathrm{ph} \mathrm{s}^{-1}$ (Cox \& Laureijs 1989), with half coming from stars in the three massive clusters. This flux is about $10 \%$ of that for the whole Galaxy, and the number of massive stars $\left(M_{\mathrm{i}}>20 \mathrm{M}_{\odot}\right)$ in the GC is about $10 \%$ of the number in the whole Galaxy. However, note that the star-formation rate in the GC is about one-hundredth of that for the whole Galaxy. Such a low star-formation rate as a function of Lyman continuum photon production necessarily follows from the relatively flat initial mass function (IMF) slope used in estimating the mass of stars formed in the young clusters. The recent star-formation history $\left(t_{\text {age }} \lesssim 50 \mathrm{Myr}\right)$ in the Galactic Center is relatively clear. Embedded H II regions trace star formation at the present time (Figer 1995, Cotera 1995, Cotera et al. 1999), while the young clusters trace star-formation that occured $2.5-5 \mathrm{Myr}$ ago. The lack of red supergiants $\left(M_{\mathrm{bol}}<-6.3 \mathrm{mag}\right.$ and $\left.M_{\mathrm{i}}>8 \mathrm{M}_{\odot}\right)$ in the region provides evidence for a very low star formation rate from 5-20 Myr ago. Indeed, a burst the size of that seen in the three clusters at $20 \mathrm{Myr}$ ago would have produced 40 red supergiants, yet we see none (other than those associated with the young clusters).

The constraints on this activity are very strong, i.e., there were fewer than $5 \times 10^{3} \mathrm{M}_{\odot}$ formed in stars over this time period, assuming the type of starformation that spawned the three massive clusters. Looking beyond $20 \mathrm{Myr}$, the picture becomes less clear because it is difficult to separate old low-mass red giants from much younger high-mass AGB stars with photometric data alone. Using spectroscopy, Blum et al. (1996) have shown that there is a relative dearth of stars with ages between 10 and $100 \mathrm{Myr}$ in the central few parsecs. Given their conclusions, I estimate a low star-formation rate during this period. These same authors, and Haller (1992), identified stars with ages on the order of a few hundred Myr in the central few arcminutes. Sjouwerman et al. (1999) identified a population of $\mathrm{OH} / \mathrm{IR}$ stars with a narrow range of expansion velocities, indicat- 

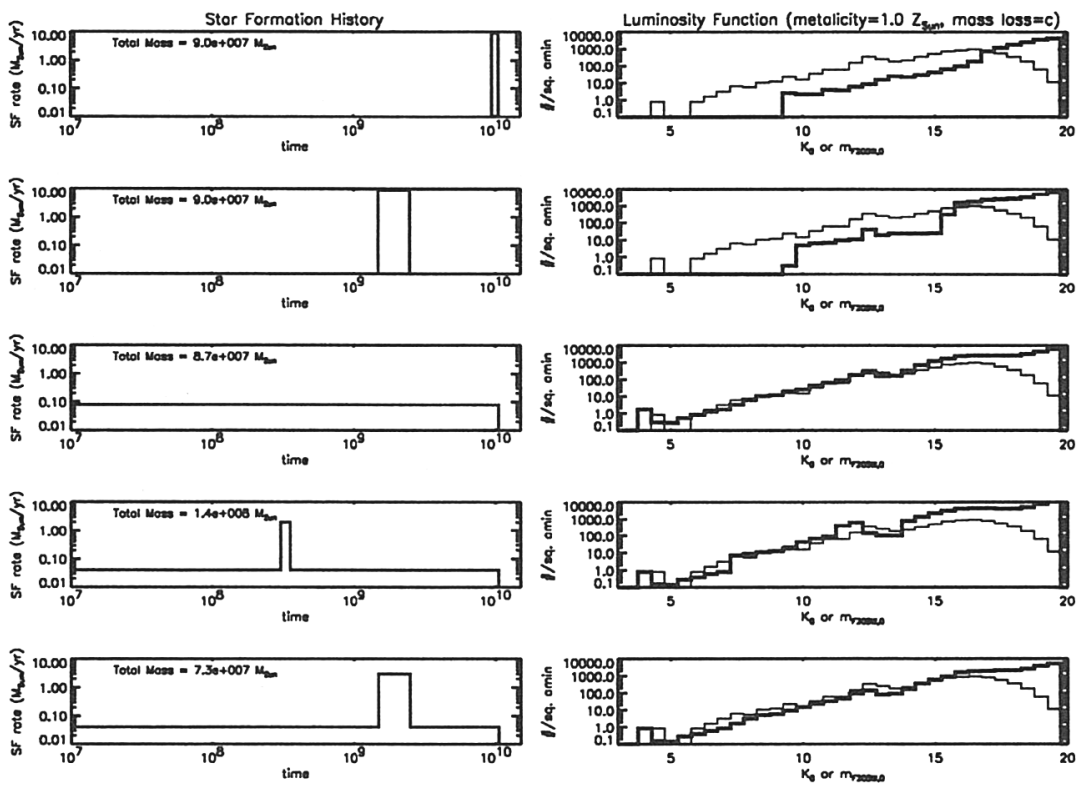

Figure 5. Model luminosity functions (right, bold) and observed luminosity functions (right, light) for various star-formation histories in the Galactic Center (left). The models use the Geneva isochrones with solar abundances and canonical mass loss rates.

ing intermediate ages and a starburst event a few Gyr ago. In addition, Frogel et al. (1999) identified an excess of bright stars in the fields they observed within 0.2 of the Galactic Center.

In order to infer the past star-formation rate, I modeled the observed surface number density of stars (Figure 4) as a function of star-formation history using the Geneva stellar evolution models, assuming a range of power-law IMFs, metallicity, and wind mass-loss rates. I considered: an ancient burst, episodic bursts, continuous formation, and combinations of these three. I used these models to produce synthetic luminosity functions for comparison with HST-NICMOS data. The surface number density in the observed luminosity functions has been set by dividing the number of stars per half magnitude bin by the area of the observations. The NICMOS fields vary in location from about $15 \mathrm{pc}$ from the GC to $55 \mathrm{pc}$, and the sample was culled of foreground and background stars by limiting the data to stars with $1.0<m_{\mathrm{F} 160 \mathrm{~W}}-m_{\mathrm{F} 205 \mathrm{~W}}<3.0\left(1.7<A_{K}<6.5\right)$. The results are presented in Figure 5.

In general, I find that the presently observed luminosity function is very well fit by continuous star-formation at the level of 0.05 to $0.1 \mathrm{M}_{\odot} \mathrm{yr}^{-1}$. The total mass of stars formed is $\sim 10^{8} \mathrm{M}_{\odot}$ in these models, although the present-day mass is less as a result of mass-loss via steady winds or supernovae.

An ancient burst model is not consistent with the presence of bright stars $\left(K_{\circ}<8.0\right)$, nor is it consistent with an enhanced brightness of the red clump (although the observational evidence for such an enhancement is controversial). 
Episodic bursts are essentially indistinguishable from continuous star-formation when using the luminosity function as a metric; clearly, one needs to examine a subset of all stars in distinguishing burst widths and periodicities. The continuous star-formation model reproduces the bright end of the luminosity function, although note that the bulk of the observed bright stars are not red supergiants, so that there must be a gap in significant star-formation activity during recent times, excepting the last $5 \mathrm{Myr}$. I find that variations in metallicity, IMF slope, or mass-loss rate, do not qualitatively affect our conclusion that continuous star-formation produces synthetic luminosity functions that best fit the observed luminosity function, compared with the other star-formation scenarios examined.

Acknowledgments. I acknowledge very useful discussions with Paco Najarro, Bob Blum, Laurant Sjouwerman, Mike Rich, Mark Morris, Sungsoo Kim, and Jay Frogel.

\section{References}

Allen, D.A., Hyland, A.R., Hillier, D.J. 1990, MNRAS 244, 706

Allen, D.A. 1994, in: R. Genzel \& A.I. Harris (eds.), The Nuclei of Normal Galaxies, NATO ASI Ser. C, Vol 445 (Dordrecht: Kluwer), p. 293

Becklin, E.E., Neugebauer, G. 1968, ApJ 151, 145

Blum, R.D., Depoy, D.L., Sellgren, K. 1995, ApJ 441, 603

Blum, R.D., Schaerer, D., Pasquali, A., Heydari-Malayeri, M., Conti, P.S., Schmutz, W. 2001, AJ 122, 1875

Cotera, A.S., Erickson, E.F., Simpson, J.P., Colgan, S.W.J., Allen, D.A., Burton, M.G. 1992, AAS 181,8702

Cotera, A.S. 1995, $\mathrm{PhD}$ Thesis, Stanford University, USA

Cotera, A.S., Erickson, E.F., Colgan, S.W.J., Simpson, J.P., Allen, D.A., Burton, M.G. 1996, ApJ 461, 750

Cotera, A.S., Simpson, J.P., Erickson, E.F., Colgan, S.W.J., Burton, M.G., Allen, D.A. 1999, ApJ 510, 747

Cox, P., Laureijs, R. 1989, in: M. Morris (ed.), The Center of the Galaxy, Proc. IAU Symp. No. 136 (Dordrecht: Kluwer), p. 121

Eckart, A., Genzel, R., Hofmann, R., Sams, B.J., Tacconi-Garman, L.E. 1995, ApJ (Letters) 445, L26

Eckart, A., Ott, T., Genzel, R. 1999, A\&A (Letters) 352, L22

Eckart, A., Genzel, R., Ott, T., Schödel, R. 2002, MNRAS 331, 917

Figer, D.F. 1995, PhD Thesis, University of California, Los Angeles, USA

Figer, D.F., McLean, I.S., Morris, M. 1995, ApJ (Letters) 447, L29

Figer, D.F., Morris, M., McLean, I.S. 1996, in: R. Gredel (ed.), The Galactic Center, ASP-CS 102, 263

Figer, D.F., Najarro, F., Morris, M., McLean, I.S., Geballe, T.R., Ghez, A.M., Langer, N. 1998, ApJ 506, 384

Figer, D.F., Becklin, E.E., McLean, I.S., et al. 2000, ApJ (Letters) 533, L49

Figer, D.F., McLean, I.S., Morris, M. 1999a, ApJ 514, 202

Figer, D.F., Morris, M., Geballe, T.R., Rich, R.M., Serabyn, E., McLean, I.S., Puetter, R.C., Yahil, A.1999b, ApJ 525, 759

Figer, D.F., Kim, S.S., Morris, M., Serabyn, E., Rich, R.M., McLean, I.S. 1999c, ApJ 525, 750 
Figer, D.F., Najarro, F., Gilmore, D., Morris, M., Kim., S.S., Serabyn, E., McLean, I.S., Gilbert, A.M., Graham, J.R., Larkin, J.E., Levenson, N.A. 2002, ApJ 581, 258

Forrest, W.J., Shure, M.A., Pipher, J.L., Woodward, C.A. 1987, in: D. Backer (ed.), The Galactic Center, AIP-CP 155, 153

Frogel, J.A., Tiede, G.P., Kuchinski, L.E. 1999, AJ 117, 2296

Geballe, T.R., Figer, D.F., Najarro, F. 2000, ApJ 530, 97

Genzel, R., Thatte, N., Krabbe, A., Kroker, H., Tacconi-Garman, L.E. 1996, ApJ 472, 153

Ghez, A.M., Kremenek, T., Tanner, A., Morris, M., Becklin, E. 2001, in: L. Kaper, E.P.J. van den Heuvel, P.A. Woudt (eds.), Black Holes in Binaries and Galactic Nuclei, ESO Astrophysics Symposia (Berlin: Springer), p. 72

Glass, I.S., Catchpole, R.M., Whitelock, P.A. 1987, MNRAS 227, 373

Haller, J.W. 1992, PhD thesis, The University of Arizona, USA

van der Hucht, K.A. 2001, New Astron. Reviews 45, 135

Krabbe, A., Genzel, R., Drapatz, S., Rotaciuc, V. 1991, ApJ (Letters) 382, L19

Krabbe, A., Genzel, R., Eckart, A., et al. 1995, ApJ (Letters) 447, L95

Lang, C.C., Goss, W.M., Rodríguez, L.F. 2001, ApJ (Letters) 551, L143

Lebofsky, M.J., Rieke, G.H., Tokunaga, A.T. 1982, ApJ 263, 736

Moneti, A., Glass, I.S., Moorwood, A.F.M. 1994, MNRAS 268, 194

Moneti, A., Stolovy, S., Blommaert, J.A.D.L., Figer, D.F., Najarro, F. 2001, A\&A 366, 106

Nagata, T., Woodward, C.E., Shure, M., Pipher, J.L., Okuda, H. 1990, ApJ 351, 83

Nagata, T., Woodward, C.E., Shure, M., Kobayashi, N. 1995, ApJ 109, 1676

Najarro, F., Hillier, D.J., Kudritzki, R.-P., Krabbe, A., Genzel, R., Lutz, D., Drapatz, S., Geballe, T.R. 1994, A\&A 285, 573

Najarro, F., Krabbe, A., Genzel, R., Lutz, D., Kudritzki, R.-P., Hillier, D.J. 1997, A\&A 325,700

Okuda, H., Shibai, H., Nakagawa, T., Matsuhara, H., Kobayashi, Y., Kaifu, N., Nagata, T., Gatley, I., Geballe, T.R. 1990, ApJ 351, 89

Paumard, T., Maillard, J.P., Morris, M., Rigaut, F. 2001, A\&A 366, 466

Rieke, G.H., Lebofsky, M.J. 1982, in: D. Backer (ed.), The Galactic Center, AIPCP 155, 194

Rieke, G.H., Rieke, M.J., 1994, in: R. Genzel \& A.I. Harris (eds.), The Nuclei of Normal Galaxies, NATO ASI Ser. C, Vol 445 (Dordrecht: Kluwer), p. 283

Salpeter, E.E. 1955, ApJ 121, 161

Scalo, J. 1998, in: G. Gilmore \& D. Howell (eds.), The Stellar Initial Mass Function, ASP-CS 142, 201

Serabyn, E., Morris, M. 1996, Nature 382, 602

Sjouwerman, L.O., Habing, H.J., Lindqvist, M., van Langevelde, H.J., Winnberg, A. 1999, in: H. Falcko, A. Cotera, W.J. Duschl, F. Melia \& M.J. Rieke, The Central Parsecs of the Galaxy, ASP-CS 186, 379

Stolte, A., Grebel, E.K., Brandner, W., Figer, D.F. 2002, A\&A 394, 459

Tamblyn, P., Rieke, G.H., Hanson, M.M., Close, L.M., McCarthy, D.W., Rieke, M.J. 1996, ApJ 456, 206

Tuthill, P.G., Monnier, J.D., Danchi, W.C. 1999, Nature 398, 487

Williams, P.M., van der Hucht, K.A., Thé, P.S. 1987, A\&A 182, 91 
Yusef-Zadeh, F., Law, C., Wardle, M., Wang, Q.D., Fruscione, A., Lang, C.C., Cotera, A. 2002, ApJ 570, 665

\section{Discussion}

KUDRITZKI: Your inventory of the Galactic Center shows only O-type stars, WR star, LBVs and RSGs. Where are the B- and A-type supergiants.?

FIGER: The clusters are not old enough to produce A-type supergiants. We do identify some early B-type supergiants, i.e., the Pistol Star.

ZINNECKER: I noted with interest that you considered the possibility of stellar mergers for the Pistol Star in the Quintuplet Cluster. Given that the average stellar density is even higher in the Arches Cluster, wouldn't you expect a stellar merger in this cluster, too? Also, can you tell us a few details of the stellar merger simulation that you showed, e.g., the initial stellar masses?

FIgER: We considered a stellar-merger scenario for the Pistol Star, after our $N$-body simulations predicted that a few mergers should have already occurred in the Arches Cluster. Our code also predicts that the Quintuplet Cluster could have been as dense as the Arches Cluster is now.

RIzzo: Several works in the millimeter range are showing that additional (unknown?) sources are nessessary to excite the Galactic Center molecular clouds. Taking it into account, do you discard the existence of other massive clusters in the GC?

FIGER: Other clusters do not exist in the central at $50 \mathrm{pc}$, as shown by a variety of near-IR and radio surveys.

MASSEY: I'm very intrigued by the result that the IMF slope of the Arches Cluster is flat. The characterics of the Arches Cluster appear to be very similar to that of R 136, where we know that the IMF has a Salpeter slope $(\Gamma \simeq-1.35)$ from my work with Hunter (1998, ApJ 493, 180). How robust is your result from IR photometry? Have you tried doing the controlled experiment and looking at R 136 (or some cluster where we know the answers) and see if you get the same result?

FIGER: Stolte et al. (2002) has measured a flat IMF in the Arches Cluster using different data than we used. We have yet to do a similar experiment for R 136 or NGC 3603 .

LANG: Don, do you have any comments about the current location (with respect to the clusters) of the molecular clouds out of which these clusters have formed?

FIgER: The cluster velocities $\left(+130 \mathrm{~km} \mathrm{~s}^{-1}\right.$ for the Quintuplet Cluster and $+50 \mathrm{~km} \mathrm{~s}^{-1}$ for the Arches Cluster) are very different from that of any nearby molecular clouds, so we believe that the natal clouds have dispersed.

HILLIER: In the Galactic Center there are constraints on the number of ionizing He I photons. Is this constraint compatible with having more than $100 \mathrm{O}$-type stars in the Galactic Center? Does it restrict the number of O3-O6 stars?

FIGER: We predict 150 O-type stars at birth, or 110 O7-B0 stars now, i.e., 4-5 Myr after birth. The collection of stars should produce a. few $10^{51} \mathrm{H}^{+} \mathrm{ph} \mathrm{s}^{-1}$ and $5 \times 10^{49}$ $\mathrm{He}^{+} \mathrm{ph} \mathrm{s}^{-1}$. Most estimates from ionized gas are lower than these numbers, but the H II regions might not capture all the emitted flux. 